\title{
Simulación de una Celda Calorimétrica mediante Elementos Finitos
}

Juan.C. Moreno (1), Jackson Ocampo (2) y Liliana Giraldo (3)

(1) Facultad de Ciencias, Departamento de Química, Grupo de Invest. en Sólidos Porosos y Calorimetría, Universidad de Los Andes, Carrera 1 No. 18 A 10, Bogotá, Colombia.

(e-mail: jumoreno@uniandes.edu.co)

(2) Facultad de Ciencias, Departamento de Física, Universidad de Los Andes, Carrera 1 No. 18 A 10, Bogotá, Colombia.

(3) Facultad de Ciencias, Departamento de Química, Universidad Nacional de Colombia, Carrera 30 calle 45, Ciudad Universitaria, Bogotá, Colombia.

\begin{abstract}
Resumen
Se ha usado el método de los elementos finitos con el fin de determinar la conveniencia de usar una forma de paralelepípedo y diferentes materiales para una celda de un microcalorímetro de conducción tipo Calvet. El empleo las condiciones de frontera en la ecuación de calor permite describir las curvas isotermas que se establecen al interior de la celda calorimétrica cuando se realiza un proceso de transferencia térmica. La existencia de puntos críticos en la geometría de la celda puede afectar la medición y ser un factor de error en la adquisición experimental de datos. La simulación permite conocer la posibilidad de disminuir estos efectos cambiando los materiales de construcción de la misma.
\end{abstract}

Palabras claves: celdas de conducción, calorímetro Calvet, ecuación de calor, elementos finitos

\section{Simulation of a Calorimetric Cell using Finite Elements}

\begin{abstract}
Finite elements have been employed in this work with the purpose of determining the convenience of using a parallelepiped geometry and different materials for a cell of a Calvet type conduction microcalorimeter. The heat equation allows to describe isotherm curves established inside the calorimetric cell during the heat transfer process. The existence of critical points in the geometry of the cell can affect the measuring process and be an factor of error during experimental data acquisition. The simulation allows to determine the feasibility of diminishing these effects by changing the construction materials.
\end{abstract}

Keywords: conduction cells, Calvet type calorimeter, heat equation, finite elements 


\section{INTRODUCCION}

Las simulaciones de elemento finito (FEHTFinite Element Heat Transfer) son una herramienta para el cálculo de diferentes fenómenos como transferencia de masa y de energía, resistencia de estructuras, control de flujo (Zienkiewicz y Taylor, 1989). El método de elementos finitos se basa en la discretización del sistema a analizar en forma de elementos poligonales o poliédricos, dependiendo si el estudio del sistema se realiza en dos o tres dimensiones. Estos elementos finitos deben tener ciertas características especiales para que el método funcione.

Las características más importantes de los elementos finitos son: tener una superficie cerrada y contenida en el sistema, un número finito de lados y forma regular.

Esta discretización del sistema se realiza colocando nodos o puntos de cálculo sobre el sistema uniéndolos con líneas de conducción (Valencia E, 1990). Se buscan formas simples para discretizar el sistema. Dividir el sistema, permite hacer una aproximación de los eventos que ocurren al interior del material de manera puntual; la idea de la discretización es reducir el área que se ha delimitado con el elemento finito a solo sus nodos componentes y a sus líneas de conducción.

Los elementos finitos están constituidos por nodos o puntos de cálculo y por líneas de conducción, tal como se muestra en la figura 1 , distribuidos en el interior del material. Estos elementos permiten hacer cálculos de conducción de calor, resistencia de estructuras y otros sobre el sistema, obteniendo información nodo a nodo de cómo cambia y evoluciona el sistema a partir de unas condiciones iniciales establecidas (Liang et al.,2005). Dependiendo de la cantidad de elementos finitos que se tengan sobre el sistema variará la precisión y la cantidad de datos que se adquieran por lo cual es necesario utilizar la mayor cantidad posible de elementos finitos para analizar un sistema, en función de los recursos computacionales disponibles. Idealmente sería conveniente hacer una discretización muy cercana al continuo. Un análogo de esta metodología podría ser el cálculo de áreas con métodos de integración discretos como Simpson o polinomios de Taylor (Fourier, 1978), en donde la cantidad de particiones en el área a calcular es la que determina la precisión del resultado.

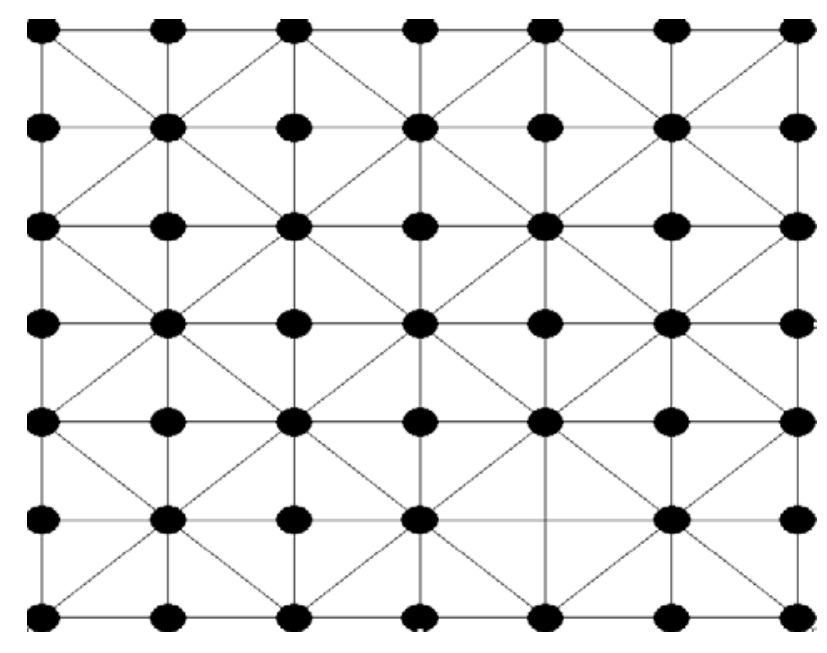

Fig 1: Nodos y Líneas de Conducción

El método de elementos finitos es una herramienta muy útil para caracterizar sistemas en los que hay transferencia de calor, ya que no solo permite analizar formas irregulares que componen el sistema sino que permite ver como evoluciona el sistema térmico en el tiempo, como se distribuye la energía a través del material y visualizar cuales son los puntos críticos en la conducción de calor. Por esta razón se realizó una simulación de elemento finito para una celda calorimétrica de un calorímetro de conducción de calor constituida por un paralelepípedo de acero inoxidable 304 con dimensiones $2 \mathrm{~cm}$ de lado en su parte externa, $6 \mathrm{~cm}$ de profundidad y paredes de $2 \mathrm{~mm}$ de espesor. Las paredes de esta celda son el objeto de simulación, dividiéndolas en elementos finitos triangulares, para así establecer por medio de los nodos y las líneas de conducción cómo sucede la transferencia de energía al interior del material.

En cada uno de los nodos del elemento finito se realizan cálculos matemáticos basados en determinadas condiciones iniciales, que determinan el comportamiento del sistema descrito por las ecuaciones de calor. Estos cálculos se realizan en cada nodo haciendo un balance energético sobre cada uno (Maskow, T. and W. Babel, 2003).

Este balance energético permite la generación ecuaciones matriciales de transferencia de energía y que su solución 
corresponda al promedio de la energía que se transmite por el sistema. Este proceso se hace computacionalmente, utilizando el programa FEHT (Karlekar y Desmond 1985). Tomando en cuenta la cantidad de nodos que se definen sobre el sistema y la cantidad de líneas de conducción que se generan seria muy dispendioso realizar los cálculos nodo a nodo de manera manual.

Este método ofrece la ventaja adicional de permitir estudiar, si es necesario, cómo es la transmisión de la energía en un sistema compuesto de muchos y diferentes materiales (Bendada A et. al 2004).

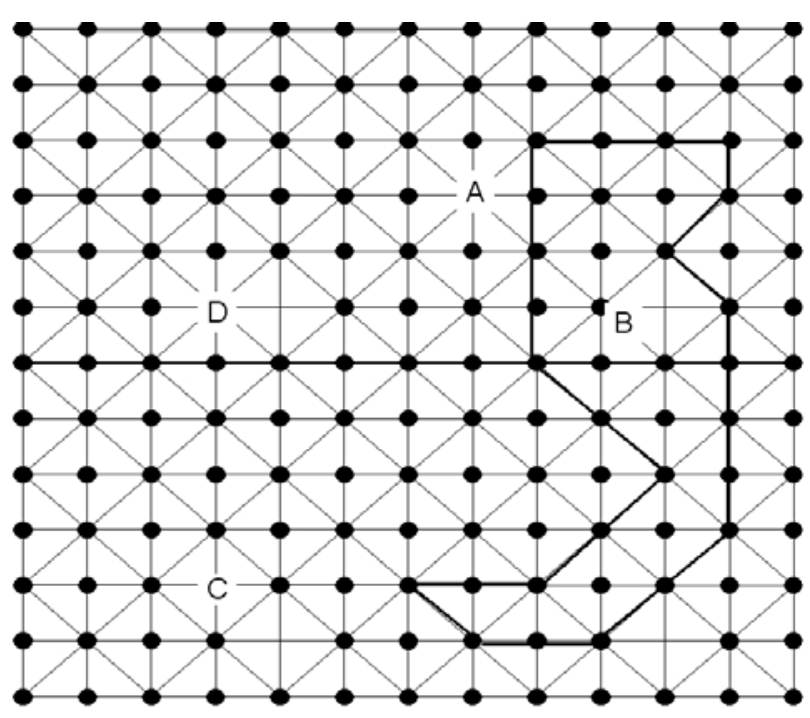

Fig 2: Distribución de elementos finitos para varios materiales contenidos en un sistema

En la figura 2 se ve una distribución de elemento finito para un conjunto de materiales contenidos en un sistema. Con diferentes colores se muestran las fronteras de los materiales y del sistema. Estas fronteras pueden tener diferentes características para permitir la transmisión de energía o ser adiabáticas. Cuando se tienen fronteras que se tocan hay que definir el flujo de energía entre una y otra. Además de la forma del sistema hay que definir los materiales de construcción y algunas de sus propiedades físicas como la densidad, el calor específico y la conductividad térmica.

\section{METODOLOGIA}

Este trabajo aborda el tema de las modelaciones de elementos finitos y su aplicación a los sistemas de transferencia de calor para caracterizar el comportamiento de una celda de un calorímetro de conducción de calor desarrollado por (Moreno, et.al 2005), ver figura 3. La idea consiste en determinar mediante subdivisión en elementos finitos cómo se transfiere la energía y cómo se distribuye al interior de las paredes de la celda para poder identificar puntos críticos de difícil conducción. Finalmente este trabajo realiza simulaciones con diferentes materiales.

La geometría de la celda es un factor importante a analizar. En este caso se tiene una celda calorimétrica en forma de paralelepípedo y resulta conveniente analizar si esta forma es la más eficiente para distribuir la energía o si tiene puntos críticos que lo dificulten.

Se puede decir que estos puntos críticos son puntos discontinuos en la geometría (Holman, 1972) de la celda, desde el punto de vista de la conducción de calor.

Resulta importante realizar una modelación de elemento finito para este sistema puesto que la precisión de un calorímetro de conducción de calor depende de cómo se esté registrando la energía circulante en el sistema y cómo esta misma energía se transmite y es absorbida por el sistema, es decir, la capacidad calorífica del calorímetro.

En el interior de esta celda calorimétrica se han realizado mediciones experimentales de conducción de calor utilizando contenidos como aire, agua, reacciones ácido base como $\mathrm{HCl}+\mathrm{NaOH}$, disoluciones de una sal como $\mathrm{KCl}$ en agua, e inmersión de carbones activados (García, 1989) en soluciones de compuestos fenólicos como el 2-4dinitrofenol, fenol, ortonitrofenol, metanitrofenol y paranitrofenol, En las figuras $4 a$ y $4 b$ se presentan ejemplos de los resultados obtenidos en dos de estas experiencias.

El modelamiento de un material mediante el uso del programa FETH consiste en definir a voluntad sus características físicas y su forma toma. Luego se definen sus líneas de conducción y sus nodos.

Luego de hacer esta definición del material, se definen las fronteras que se encuentran en contacto, es decir, el lugar donde ocurren las transferencias de energía. 

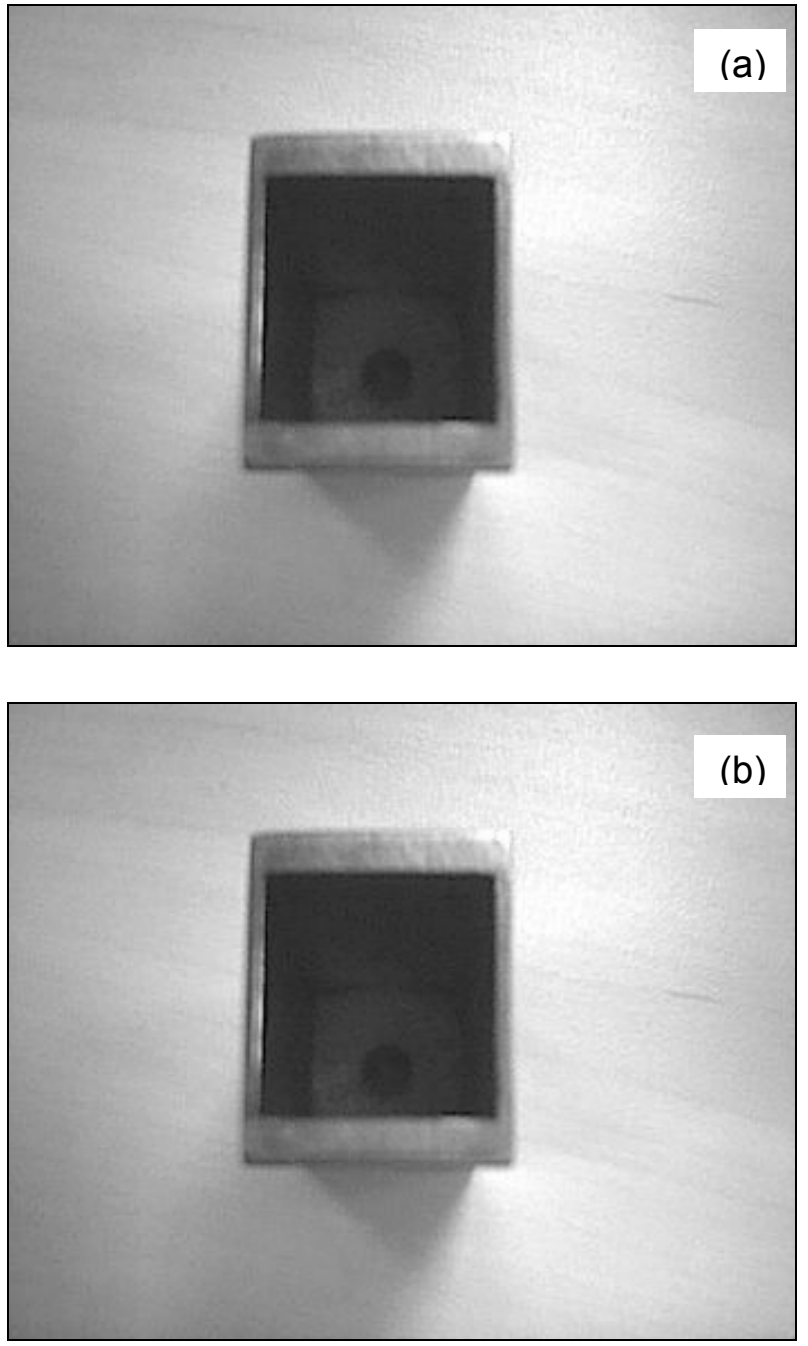

Fig 3: a) Vista superior de la celda Calorimétrica. b) Vista lateral de la celda Calorimétrica montada en el calorímetro.

La simulación permite conocer como se definen los contornos de temperatura en el interior del material, es decir, las curvas isotérmicas y así ver en qué direcciones se transfiere la energía en mayor cantidad (gradientes de temperatura).

Se tomara un corte transversal de la celda calorimétrica que se muestra en la figura 3 y se modelara en dos dimensiones, dado que las observaciones que se logren en dos dimensiones se pueden generalizar a tres simplemente considerando que la celda esta compuesta por capas de espesor infinitesimal adyacentes.

Experimentalmente se ha trabajado con un calorímetro de conducción el cual consta de una celda calorimétrica, un difusor de calor por conducción, de aluminio, con cuatro termopilas en serie incrustadas.
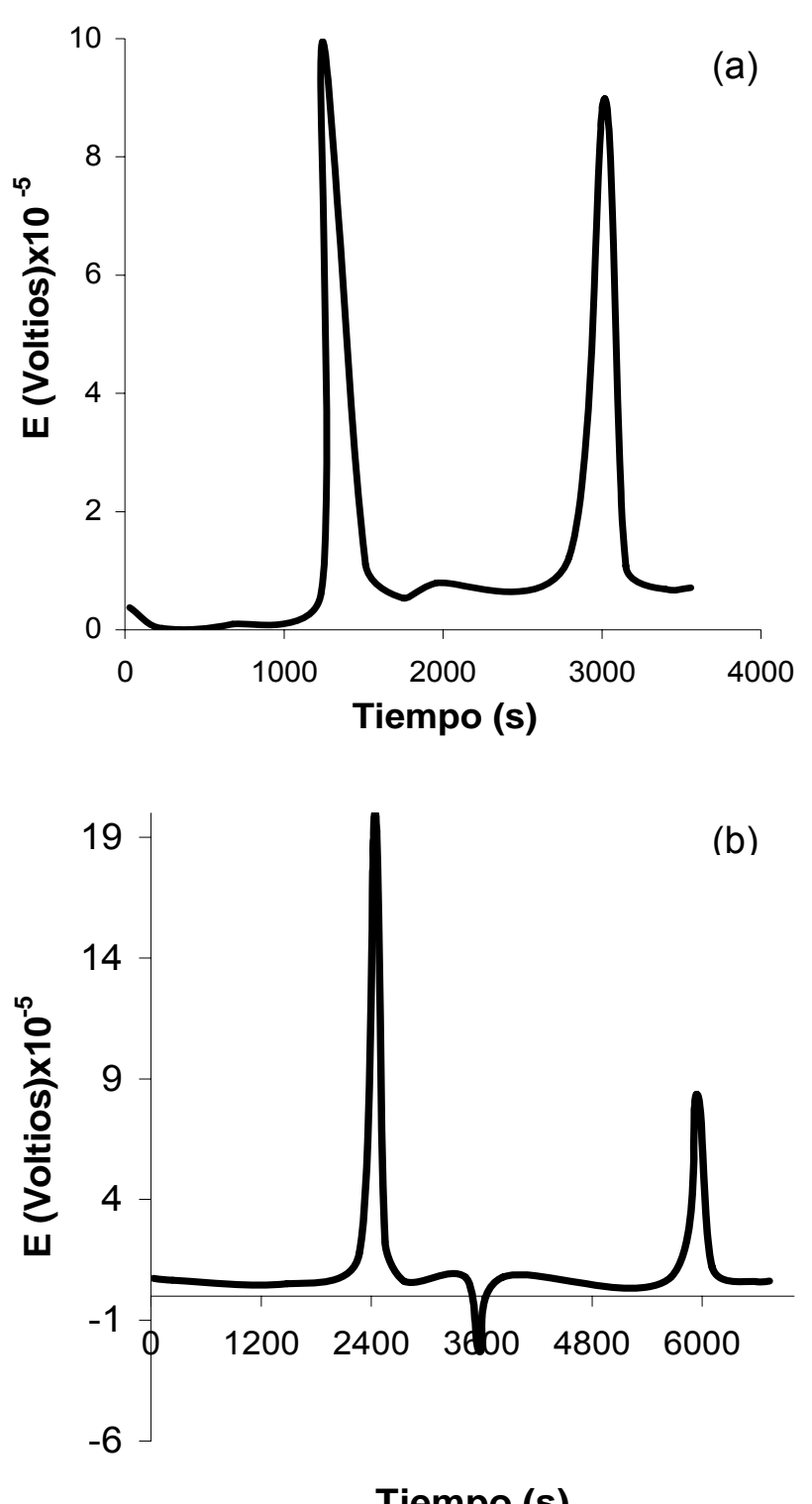

Fig 4:a) Termo! (b) a de Fenol adsorbido en carbón activadı ermograma de 2-4 Dinitro Fenol adsorbido en carbón activado

El procedimiento de operación del calorímetro es el siguiente:

1. Se coloca en la celda calorimétrica un líquido, por ejemplo agua.

2. Se coloca la celda en el interior del contorno difusor de conducción.

3. En una ampolleta de vidrio se coloca el material adsorbente, el reactivo o el soluto, según sea el caso. Esta ampolleta se sujeta en un porta ampolletas que se encuentra en la tapa del calorímetro, en la cual adicionalmente se encuentra una resistencia de $100 \Omega$, con la cual se realiza una la calibración eléctrica después de cada medida.

Información Tecnológica - Vol. 18 Nº 2 - 2007 
4. Se cierra el sistema y se espera que llegue a equilibrio térmico.

5. Se rompe la ampolleta con lo cual comienza la liberación de energía, ya sea de absorción, de reacción o de solución.

6. Terminada la liberación de energía se espera que el sistema llegue de nuevo al equilibrio y se somete a una diferencia de potencial conocida, para obtener un pico de cambio de temperatura de calibración mediante el trabajo eléctrico efectuado

El modelo de simulación que se utilizó es un método de ecuaciones diferenciales finitas. El programa FETH se basa en la ecuación diferencial de calor (Ecuación 1) para determinar como va cambiando la temperatura en cada uno de los nodos en la estructura, a medida que avanza el proceso de transmisión. Se obtienen así las curvas isotérmicas (contornos de temperatura) en el interior del material

$\frac{\partial}{\partial x}\left(k \frac{\partial T}{\partial x}\right)+\frac{\partial}{\partial y}\left(k \frac{\partial T}{\partial y}\right)+\dot{q}+\rho c \frac{\partial T}{\partial y}=0$

La ecuación (1), en el método del elemento finito, se convierte en una expresión integral que pueda dar una solución aproximada de ella. Esto se logra multiplicando todo por una función arbitraria $f(x, y)$ e integrando sobre el área $A$ :

$$
\iint_{A} f(x, y)\left[\frac{\partial}{\partial x}\left(k \frac{\partial T}{\partial x}\right)+\frac{\partial}{\partial y}\left(k \frac{\partial T}{\partial y}\right)+\dot{q}+\rho c \frac{\partial T}{\partial y}\right] d x d y=0
$$

la solución exacta $T(x, y, t)$ sería aquella que hace el término entre paréntesis cuadradas igual a cero, para todo $\mathrm{x}$, y y t. Las soluciones aproximadas hacen que la integral sea cero solo para puntos seleccionados dentro del área $\mathrm{A}$.

La resolución de la ec (2) comienza integrando los dos primeros términos $y$ reorganizando para llegar a:

$$
\begin{array}{r}
\frac{d}{d t} \iint_{A} f \rho c T d x d y+\iint_{A} f(x, y)\left[\frac{\partial f}{\partial x} k \frac{\partial T}{\partial x}+\frac{\partial f}{\partial y} k \frac{\partial T}{\partial y}\right] d x d y= \\
=\iint_{A} f \dot{q} d x d y-\int_{B} f q_{n}{ }_{n} d s
\end{array}
$$

donde $q_{n}{ }_{n}$ es el flujo calorífico local que entra a $A$ a través de la frontera $B$.

$B$ se puede abrir en $B=B_{c}+B_{s}+B_{a}+B_{t}$ donde las porciones de la frontera corresponden $\mathrm{a}: \mathrm{B}_{\mathrm{c}}$ porción convectiva para la cual $q_{n}{ }_{n}=h\left(T_{\infty}-T\right)$. $B_{s}$ porción con flujo de calor tal que $\mathrm{q}_{\mathrm{n}}=\mathrm{q}_{\mathrm{s}}$. $\mathrm{B}_{\mathrm{a}}$ porción para la cual $\mathrm{q}_{\mathrm{n}}=0$ y $\mathrm{B}_{\mathrm{t}}$ porción de la frontera con temperatura especificada. Esto permite considerar explícitamente las condiciones de frontera:

$$
\begin{aligned}
\frac{d}{d t} & \iint_{A} f \rho c T d x d y+\iint_{A} f(x, y)\left[\frac{\partial f}{\partial x} k \frac{\partial T}{\partial x}+\frac{\partial f}{\partial y} k \frac{\partial T}{\partial y}\right] d x d y= \\
& =\iint_{A} f \dot{q} d x d y-\int_{B C} f h\left(T_{\infty}-T\right) d s+\int_{B S} f q_{S}^{\prime \prime} d s+\int_{B t} f q_{n}^{\prime \prime} d s
\end{aligned}
$$

En este punto ya se hace necesario escoger $f$ tal que valga cero a una cierta temperatura de la frontera, haciendo que la última integral en (4) valga cero.

En el método del elemento finito se aproxima la frontera $B$ a nodos conectados por segmentos rectos con lo que la frontera real se reemplaza por una aproximación poligonal. $\mathrm{Se}$ pueden hacer conexiones adicionales entre los nodos para llegar a definir un conjunto de elementos triangulares. Se hace la suposición de que la temperatura dentro del triángulo varía linealmente entre los valores que tiene en los vértices nodales. El problema es pues determinar la temperatura en cada nodo. La función $T(x, y, t)$ se puede aproximar por un vector de $\mathrm{n}$ temperaturas y como cada temperatura en el punto $(\mathrm{x}, \mathrm{y})$, dentro del elemento triangular, varía linealmente entre la temperatura de los vértices, puede decirse que en cualquier punto dentro del elemento la temperatura es

$$
\begin{aligned}
& T(x, y, t)=w_{1}(x, y) T_{1}(t)+w_{2}(x, y) T_{2}(t)+\ldots . .+ \\
& +w_{n}(x, y) T_{n}(t)=w^{\top}(x, y) t(t)
\end{aligned}
$$

donde $T_{i}(t)$ es la temperatura del vértice i en el tiempo $t$ y $w_{i}(x, y)$ es la función ponderadora (weighting) para el nodo i, que vale 1 en el nodo i y 0 en todos los demás y varía de 0 a 1 a lo largo de las fronteras opuestas al nodo i. La notación matricial debe ser entendida como 


$$
\mathbf{w}^{\top}=\left[\begin{array}{llll}
\mathbf{w}_{1} & \mathbf{w}_{2} & \ldots . & \mathbf{w}_{\mathrm{n}}
\end{array}\right] \quad \mathbf{y} \quad \mathbf{t}=\left[\begin{array}{l}
T_{1} \\
T_{2} \\
\vdots \\
T_{n}
\end{array}\right]
$$

Las funciones $\mathrm{w}$ se encuentran para cada elemento "e" resolviendo tres ecuaciones simultáneas de la forma :

$$
\mathrm{T}(\mathrm{e})=\mathrm{c}_{1}{ }^{(\mathrm{e})}+\mathrm{c}_{2}{ }^{(\mathrm{e})} \mathrm{x}+\mathrm{c}_{3}{ }^{(\mathrm{e})} \mathrm{y}
$$

para $\mathrm{c}_{1}{ }^{(\mathrm{e})}, \mathrm{c}_{2}{ }^{(\mathrm{e})}, \mathrm{c}_{3}{ }^{(\mathrm{e})}$, en términos de las temperaturas de los vértices. La función w para el nodo $\mathrm{i}$ en las coordenadas $(\mathrm{x}, \mathrm{y})$ dentro del elemento e, compuesto por los nodos i, j, k es :

$w_{i}^{(e)}(x, y)=\frac{1}{\left(x_{i j} y_{j k}-x_{j k} y_{i j}\right)}\left[x_{j} y_{k}-x_{k} y_{j}-x y_{j k}-x_{j k} y\right]$

donde $x_{i j}$ es la abreviación de $x_{j}-x_{i}$. Ahora ya es posible determinar, usando (5), la distribución de temperaturas nodales. El paso siguiente es reemplazar $\mathrm{T}$ en (4) por la expresión (7) y escoger, por conveniencia, la arbitraria $f_{i}(x, y)$ como idéntica a $w_{i}(x, y)$, para obtener :

$\left[\iint_{A} \mathrm{w} \rho c \mathrm{w}^{T} d x d y\right] \frac{d \mathrm{t}}{d t}+\left[\iint_{A} f(x, y)\left[\frac{\partial \mathrm{w}}{\partial x} k \frac{\partial \mathrm{w}^{T}}{\partial x}+\frac{\partial \mathrm{w}}{\partial y} k \frac{\partial \mathrm{w}^{T}}{\partial y}\right] d x d y\right] \mathrm{t}+$
$+\left[\int_{B C} \mathrm{w} \mathrm{w}^{T} d s\right] \mathrm{t}=\quad \iint_{A} \mathrm{w} q d x d y-\int_{B C} \mathrm{w} h T_{\infty} d s+\int_{B S} \mathrm{w} q_{S}^{\prime \prime} d s$

Con base en lo anterior el programa construye las matrices de transferencia térmica:

$$
\mathrm{Ct}+(\mathrm{K}+\mathrm{H}) \mathrm{t}=\mathrm{g}+\mathrm{h}+\mathrm{q}
$$

$t$ es el vector de temperaturas en cada nodo, $t$ la derivada temporal de $\mathrm{t}, \mathrm{C}$ la matriz capacitancia (involucra el peso y el calor específicos), K la matriz de conducción (incluyendo la conductividad térmica), $\mathrm{H}$ la matriz de convección (con el coeficiente de transferencia de calor en la frontera), $\mathrm{g}$ el vector de generación, $h$ el vector de convección (involucra el coeficiente de transferencia de calor especificado y la temperatura de la frontera), q el vector de flujo calorífico (especificado en la frontera).
Para dar significado de energía a los vectores se puede re-arreglar (9):

$\mathrm{Ct}+\mathrm{Kt}-\mathrm{g}=(\mathrm{h}-\mathrm{Ht})+\mathrm{q}$

Ct es la rata de almacenamiento de la energía en cada sistema nodal, Kt es la rata total de conducción de calor de cada sistema nodal a los alrededores, $g$ es el vector de generación de energía en cada elemento nodal, $\mathrm{h}-\mathrm{Ht}$ vector de convección especificada que entra en cada elemento, q el vector con el flujo calorífico especificado que entra en cada elemento nodal.

La ecuación (10) se puede dividir en dos partes:

$q_{o}=C t+K t-g$
$q_{o}=(h-H t)+q$

para obtener dos expresiones para calcular las entradas de energía del exterior de la región a cada uno de los sistemas nodales.

La ecuación (11) explicita el balance en cada elemento según el cual la energía que entra más la energía generada debe ser igual al almacenamiento más la conducción hacia el exterior del elemento. En el caso que nos ocupa resulta conveniente usar la ecuación (11), con $\mathrm{g}=0$ :

$\mathrm{q}_{\mathrm{o}}=\mathrm{Ct}+\mathrm{Kt}$

para generar las simulaciones que nos ocupan.

\section{MODELACION}

Se establecen los parámetros necesarios en la ejecución del programa con las propiedades físicas del material de la celda calorimétrica (acero inox 304): densidad a 20 ${ }^{\circ} \mathrm{C}, 7.9 \frac{\mathrm{Kg}}{\mathrm{dm}^{3}}$, conductividad térmica $15 \frac{\mathrm{W}}{\mathrm{mK}}$, y el calor especifico a $20^{\circ} \mathrm{C}, 500 \frac{\mathrm{J}}{\mathrm{KgK}}$. Luego se procede a la diagramación computacional de la celda, y a su posterior división en elementos finitos, que para este caso en particular consiste en generar triángulos rectángulos al interior del material, y tres líneas de nodos en la parte interna del material como se observa en la figura 5 . Por último se definen los materiales que están en contacto, que son el acero 304 en la parte 
externa y paredes y aire en el interior el aire. La misma simulación se corrió con agua en el interior.

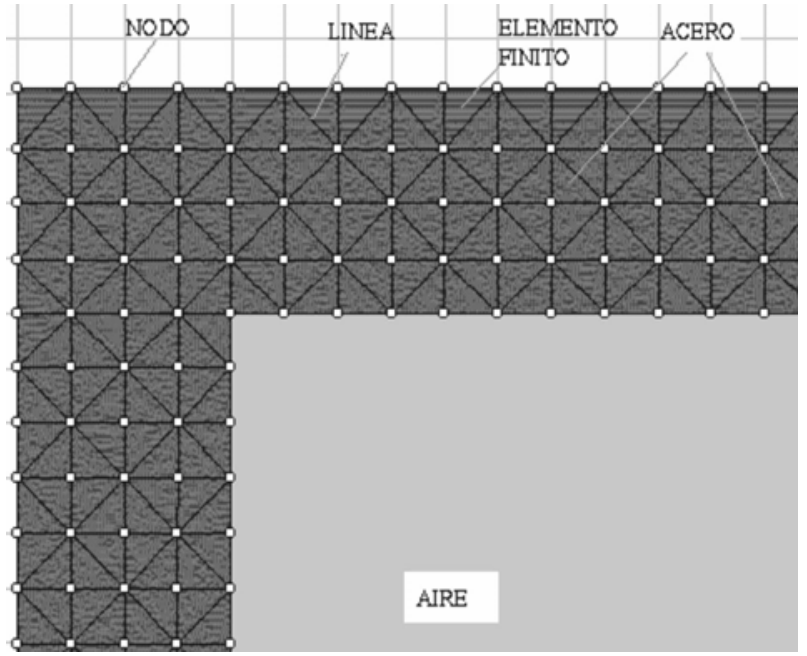

Fig 5: Distribución de elementos finitos y triangulación

La condición inicial en la simulación es el estado en el cual el fluido esta a una temperatura mayor que la celda, sin generación adicional de calor. Se da inicio a la simulación para obtener las curvas isotérmicas generadas en el cálculo que a su vez dan los gradientes de temperatura, así como el tiempo necesario para que el sistema llegue al equilibrio térmico o a un estado estacionario de conducción de calor, en el cual los gradientes no cambien más

Los valores de temperatura inicial que se utilizaron para realizar la simulación son: en la fronteras externas de la celda y en el interior de la pared $23{ }^{\circ} \mathrm{C}$, en la frontera interna del material y en el fluido (agua o aire) contenido en la celda $23.05{ }^{\circ} \mathrm{C}$. La diferencia de temperatura entre el interior y el exterior de la celda es de tan solo $0,05^{\circ} \mathrm{C}$ y corresponde al orden de magnitud de las ocurrencias y necesidades experimentales.

\section{RESULTADOS}

Los datos gráficos obtenidos con el simulador se muestran en la figura 6 . Se observa el proceso de conducción de calor en el interior de las paredes de la celda calorimétrica. La intensidad del color rojo indica la temperatura. La mayor intensidad corresponde a $23,05^{\circ} \mathrm{C}$.

En la figura $6 a$ se representa la condición inicial del sistema. En la figura $6 \mathrm{~b}$ comienza el proceso de transferencia de calor que conducirá al estado estacionario (en algunos casos podrá ser al estado de equilibrio). Se observa que los contornos de temperatura se mueven y cambian de manera uniforme en casi todo el material. En este "casi" empieza a verse la influencia de la geometría del cuerpo, de sección transversal cuadrada, en la conducción de calor.

Como los cambios en el gradiente térmico ocurren de manera similar en todos los vértices se muestra tan solo un vértice.

En la figura 6c. Se observa una ampliación de la esquina superior izquierda de la celda en la que aparece un sesgo muy marcado indicando un punto de difícil conducción de calor. Posteriormente en las figuras $6 \mathrm{~d}$ y $6 \mathrm{e}$ se observa que en las paredes del material la temperatura tiende a aumentar de manera uniforme $y$ al ver los gradientes de temperatura de la figura $6 f$ se muestra el punto frío causado por la geometría de la celda. En esta esquina no se establece un gradiente de temperatura por lo cual el flujo calorífico es desviado. Esta situación no se modifica hasta la estabilización del sistema, en las figuras $6 \mathrm{k}$ y $6 \mathrm{l}$.

Otro de los resultados que se obtiene del simulador es el flujo de calor proporcional al gradiente de temperatura $\left(\frac{{ }^{\circ} \mathrm{C}}{\mathrm{m}}\right)$. Al principio de la interacción el gradiente aumenta y cuando se llega a la estabilización el gradiente y el flujo se hacen menores. Inicialmente se tiene un valor de $46.5 \quad \frac{{ }^{\circ} \mathrm{C}}{\mathrm{m}}$ y en el estado estacionario el gradiente tiene un valor de $0.45 \frac{{ }^{\circ} \mathrm{C}}{\mathrm{m}}$. Este gradiente residual existe debido a que en el modelo computacional la energía no se transmite mas allá de las paredes de la celda, es decir que no se programó un contorno conductor en el cual se transmita la energía térmica hasta otra frontera adiabática.

Para poder entender el comportamiento del sistema en sus vértices es necesario ver las curvas isotérmicas (ver figura 7) que se forman en el interior de la pared de la celda y observar como el calor se distribuye por el material.

En la figura 7 se presentan las curvas isotérmicas que permiten analizar de una manera mas detallada el como influye la geometría del cuerpo, En la figura 7a vemos 

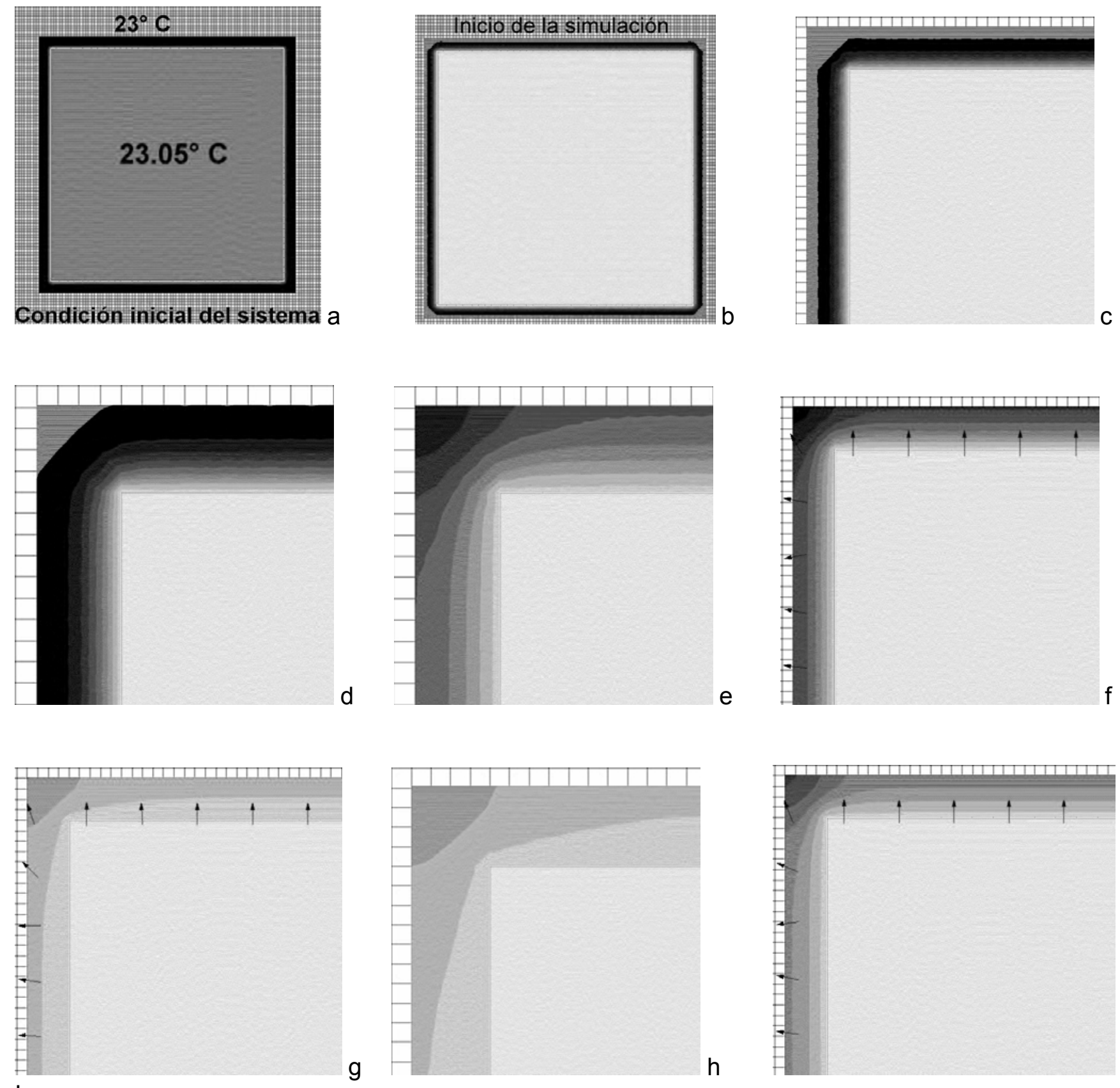

I
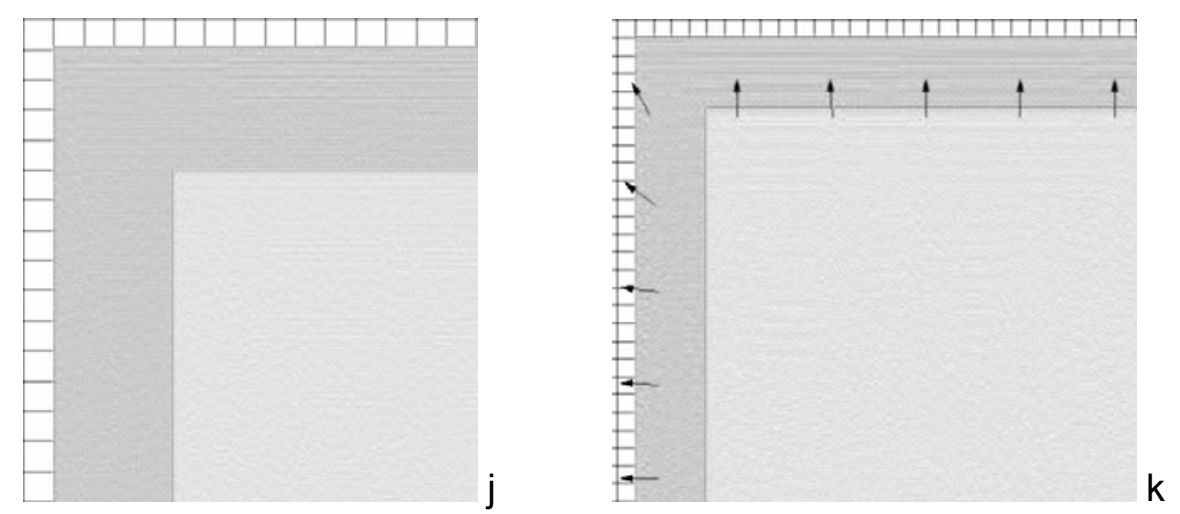

Fig. 6: Secuencia de resultados gráficos entregados por el simulador. 
que hay una distribución muy uniforme de las líneas isotérmicas en el interior del material, esto ocurre en al mismo tiempo en que se generan los contornos de temperatura vistos en la figura $6 \mathrm{~b}$. Luego, a medida que el sistema evoluciona en el tiempo, las líneas isotérmicas se mueven de manera muy notoria y se ven muy juntas, figura $7 b$ que corresponde al mismo tiempo de la figura $6 \mathrm{~d}$. Se aprecia de manera especial el punto discontinuo de conducción. Por él pasa una línea isotérmica que se encuentra muy alejada del conjunto de curvas. Esto muestra que el gradiente de temperatura es menor, siéndolo también el flujo calorífico perpendicular a él, así este punto permanece frío. De la figura 7c en adelante se ve como el punto discontinuo genera una sección casi triangular en las isotermas que pasan por la mitad más externa de la esquina de la celda. En él interior de esta sección triangular las curvas isotérmicas forman parábolas, lo cual indica que entre más cerca del punto discontinuo se esté será más difícil el flujo de calor hacia él.

Se cambió el material y el contenido de la celda con el objetivo de ver si los materiales influían en el comportamiento descrito y se encontró que el material de la celda ni su contenido influyen en el comportamiento de los puntos discontinuos Sin embargo se observó influencia en el tiempo necesario para llegar a la estabilización del gradiente así como en el valor de la temperatura de estabilización. Este aspecto es muy interesante ya que permite identificar cuál es el material más eficiente con respecto al tiempo transitorio en la conducción de calor y cual es el que disminuye las pérdidas indeseadas de calor.

En la tabla 1 se observan estos resultados Hay dos aspectos que llaman la atención;
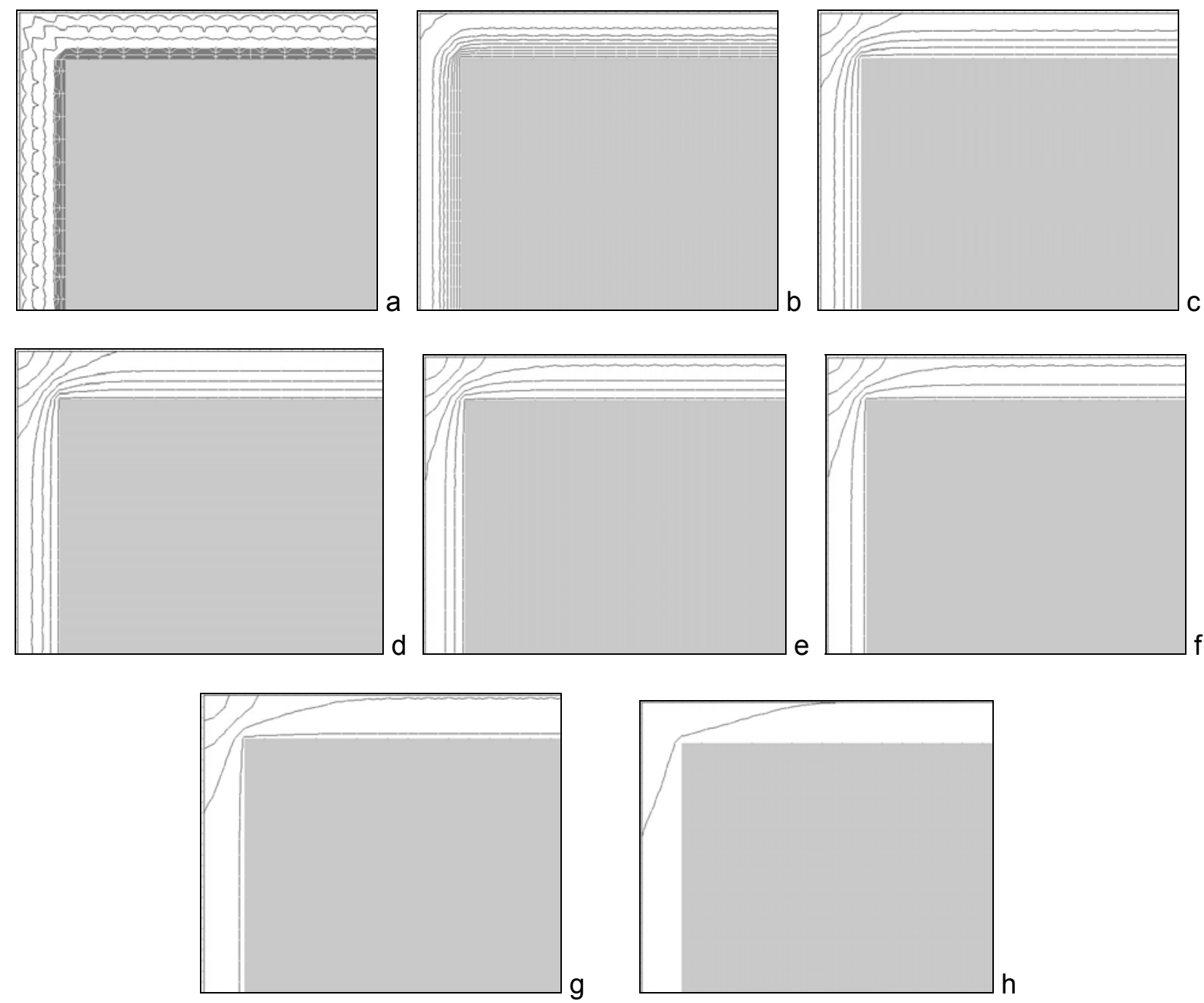

Fig. 7: Consecutivo de curvas isotérmicas dentro del sistema. 
Tabla 1: datos de temperatura $\mathrm{T}$ en ${ }^{\circ} \mathrm{C}$ y tiempo t en segundos para diferentes materiales del interior y el exterior de la celda

\begin{tabular}{|c|c|c|c|c|}
\hline Int. Celda $\backslash$ Mat. Celda & Acero 304 & Aluminio & Cobre & Plata \\
\hline \multirow{2}{*}{ Aire } & $\mathrm{T}=23.039$ & $\mathrm{~T}=23.041$ & $\mathrm{~T}=23.043$ & $\mathrm{~T}=23.045$ \\
& $\mathrm{t}=0.060$ & $\mathrm{t}=0.020$ & $\mathrm{t}=0.009$ & $\mathrm{t}=0.009$ \\
\hline \multirow{2}{*}{ Agua } & $\mathrm{T}=23.039$ & $\mathrm{~T}=23.041$ & $\mathrm{~T}=23.043$ & $\mathrm{~T}=23.045$ \\
& $\mathrm{t}=0.060$ & $\mathrm{t}=0.020$ & $\mathrm{t}=0.009$ & $\mathrm{t}=0.009$ \\
\hline
\end{tabular}

Tabla 2: Conductividad térmica y calor específico de los materiales simulados mas el vidrio

\begin{tabular}{|l|c|c|c|c|c|}
\hline & Acero 304 & Aluminio & Cobre & Plata & Vidrio \\
\hline Conductividad (w/m K) & 15 & 204 & 385.9 & 429 & 0.745 \\
\hline Cp (KJ/kg.K) & 0.45 & 0.92 & 0.38 & 0.23 & 0.60 \\
\hline
\end{tabular}

- No hay diferencia entre los datos tomados con aire y con agua en el interior.

- El tiempo de establecimiento del estado estacionario del cobre y de la plata es el mismo.

El primer aspecto se explica teniendo en cuenta la definición de las condiciones de simulación donde se propuso que no hubiera generación de calor al interior de la celda, pero que estuviera a una temperatura mayor que el exterior. En el interior se tiene un fluido ideal en el que todas sus partículas tienen la misma energía cinética, eso quiere decir que cualquier fluido que se coloque al interior de la celda con esas condiciones no modificará el sistema. En experimentos reales con un par de fluidos, por ejemplo agua en estado liquido y aire, se sabe que sus capacidades caloríficas son diferentes, y que la cantidad de moléculas contenidas en el volumen es diferente, así que aunque los dos fluidos estén a la misma temperatura la cantidad de partículas que interactúan son diferentes, y esto cambia radicalmente las mediciones calorimétricas.

Con respecto al segundo punto es probable que el tiempo de estabilización de los gradientes no sea el mismo para la plata y el cobre, lo que ocurre es que el programa no tiene sensibilidad suficiente para hacer la distinción. Al observar la simulación se puede apreciar notablemente que la plata es más rápida que el cobre en la conducción de calor, Esta es una visualización del efecto de la diferencia en el coeficiente de conducción térmica. En la tabla 2 se encuentran los coeficientes de conducción térmica y los calores específicos de los materiales usados en la simulación.

\section{CONCLUSIONES}

La sección transversal cuadrada de la celda presenta puntos discontinuos en el comportamiento de los gradientes de temperatura que pueden afectar la medición calorimétrica ya que las curvas isotermas no se reparten uniformemente en el material. El acero utilizado 304 no es un eficiente transmisor del calor. Por constantes (ver tabla 2) está más cerca del vidrio que de cualquiera de los otros metales.

El tiempo de estabilización reportado (entre 0.009 y $0.06 \mathrm{seg}$ ) es el necesario para estabilizar los gradientes, luego, el sistema, en estado estacionario, debe transferir durante unos 100 a 300 seg el calor contenido en el interior de la celda (ver figuras $4 a$ y $4 b)$.

Entre los materiales simulados la plata es el material de construcción más conveniente, desde el punto de vista de la conducción de calor.

Así mismo se puede concluir que la celda de sección transversal cuadrada se puede usar para las mediciones calorimétricas teniendo en cuenta que hay lugares en los cuales las isotermas no se reparten de manera uniforme donde no resulta conveniente ubicar sensores en el exterior 
de las paredes para medir efectos térmicos producidos en el interior de la celda. Existen regiones a lo largo de las paredes de celda en donde las curvas isotermas no son afectadas por la geometría constituyéndose en excelentes puntos de medición. Sin lugar a dudas, desde este punto de vista, usar una celda cilíndrica sería más adecuado.

Uno de las cualidades fundamentales de una celda calorimétrica es que transfiera muy bien el calor y que no intervenga en el proceso térmico que se esta midiendo, por lo cual el acero es la mejor opción dada su alta pasividad química. Si se utilizara una celda de plata o aluminio para medir, por ejemplo, la energía liberada por una reacción ácido base, es muy posible que el material de la celda reaccione y haga parte del fenómeno alterando drásticamente la medición calorimétrica. El inconveniente de usar acero inoxidable 304 reside en el hecho de que sólo para establecer los gradientes (calentar la celda) se produce una caída en la temperatura de $0,05 \mathrm{C}$ a $0.039 \mathrm{C}$, es decir $0.011 \mathrm{C}$.El vidrio produciría aproximadamente una disminución del doble, lo que representa una pérdida en la lectura de la temperatura de cerca del $44 \%$. Esto sin contar la pérdida que sucedería en la interfase con el elemento sensor y en el elemento sensor mismo. Estos materiales deben elegirse sólo en casos de necesidad de inercia química a bajo costo.

\section{AGRADECIMIENTOS}

Los autores agradecen al convenio marco existente entre la Universidad Nacional de Colombia y la Universidad de Los Andes. Al Proyecto Semilla del Fondo de Investigaciones, Facultad de Ciencias de la Universidad de Los Andes, Colombia.

\section{REFERENCIAS}

Bendada A. et al., Characterization of thermal contact in injection molding via the combination of an infrared hollow waveguide system and a two thermocouple probe, 16th World Conference on Nondestructive Testing, Montreal, Canada, August 30 September 3, CD-ROM Proceedings, (2004).
Fourier, J., The Analitical Theory of Heat, $1^{\text {a }}$ Edition, 345-365. Dover Publications Inc, New York, USA (1978).

García, J. M., Macro Elementos Finitos En Transferencia De Calor, Tesis de pregrado, Departamento de Ingeniería Mecánica, Universidad de los Andes, Bogota, Colombia (1989)

Holman, J.P., Transferencia De Calor, 2a Edición 23-87. McGraw Hill, New York USA (1972).

Karlekar, B.V., y Desmond, R. M., Transferencia De Calor, $2^{a}$ edition, 245-257. Interamericana, Ciudad de México, México (1985).

Liang et al. Cure Characterization of Pultruded Soy-based Composites., Journal of Reinforced Plastics and Composites. 24: 1509-1520 (2005)

Maskow, T. and W. Babel. Thermokinetic description of anaerobic growth of Halomonas halodenitrificans using a static microcalorimetric ampoule technique. J. Biotechnol. 101: 267-274 (2003)

Moreno, J.C,, L. Giraldo, E. Vargas y P. Martinez, Isoperibolic calorimetric cell with electronic integrator for temperature measurement, Instrumentation Science \& Technology, 33(1), 61-71 (2005).

Valencia, E., Macro Elementos Dinámicos En Transferencia De Calor, Tesis de pregrado, Departamento de Ingeniería Mecánica, Universidad de los Andes, Bogota, Colombia (1990)

Van Steenberge $\mathrm{N}$. et al., Analytical and Finite Element Models of the Thermal Behavior for Lead-free Soldering Processes in Electronic Assembly.(2005), http://www.imec.be/ALSHIRA/ Acceso: 17 de September (2006)

Zienkiewicz, O.C. y Taylor, R.L., The Finite Element Method, $4^{\text {a }}$ edition, Volume I, 206287. M Graw Hill, New York, USA (1989). 